\title{
El puente Doménico Parma (Colombia): alternativas en el diseño de una estructura suspendida
}

\section{Alternatives in the design of a suspension structure: The Doménico Parma Bridge (Colombia)}

$\underline{\text { Camilo Villate }}^{(*)}$, Nicolás Parra ${ }^{(* *)}$, Jorge Galindo-Díaz ${ }^{(* * *)}$

\section{RESUMEN}

Este artículo describe y analiza la manera en que el ingeniero Doménico Parma desarrolló el proceso de diseño del puente suspendido sobre el río Chinchiná (Colombia), caracterizado por tener un mástil único y vertical, con forma de diapasón, situado en el punto medio del tablero vehicular que soporta. A partir del reconocimiento de las diferentes alternativas estudiadas previamente por Parma, se demuestra la manera en que la solución finalmente adoptada logra conciliar aspectos de naturaleza mecánica con la forma de la estructura.

Palabras clave: Innovación estructural, puente suspendido, diseño estructural, ingeniería colombiana.

\section{ABSTRACT}

This article describes and analyzes the way in which the engineer Doménico Parma developed the design process of the suspension bridge over the Chinchiná River (Colombia), characterized by having a unique and vertical mast, shaped like a tuning fork, located at the midpoint of the board vehicular that it supports. Based on different alternatives previously studied by Parma, the final solution manages to reconcile aspects of a mechanical nature with the form of the structure.

Keywords: Structural innovation, suspension bridge, structural design, Colombian engineering.

(*) Arquitecto. MSc. Profesor Asociado. Universidad de los Andes, Bogotá (Colombia).

(**) Ingeniero Civil. MSc. Profesor Asociado. Universidad de los Andes, Bogotá (Colombia).

$\left.{ }^{* * *}\right)$ Arquitecto. PhD. Profesor Titular. Universidad Nacional de Colombia, Manizales (Colombia).

Persona de contacto/Corresponding author: cvillate@uniandes.edu.co (C. Villate).

ORCID: https://orcid.org/oooo-0002-3097-2009 (C. Villate); https://orcid.org/oooo-0001-9668-167X (N. Parra);

https://orcid.org/oooo-0oo1-8407-8347 (J. Galindo-Díaz).

Cómo citar este artículo/Citation: Villate, Camilo; Parra, Nicolás; Galindo-Díaz, Jorge (2020). El puente Doménico Parma (Colombia): alternativas en el diseño de una estructura suspendida. Informes de la Construcción, 72(559): e354. https://doi.org/10.3989/ic.72226.

Copyright: (C) 2020 CSIC. Este es un artículo de acceso abierto distribuido bajo los términos de la licencia de uso y distribución Creative Commons Reconocimiento 4.0 Internacional (CC BY 4.0). 


\section{INTRODUCCIÓN}

El trabajo en Colombia del ingeniero italiano Doménico Parma ha sido escasamente estudiado a pesar de la gran cantidad de proyectos en los que participó a lo largo de sus 45 años de trabajo profesional en Colombia, aplicando en muchos de ellos procesos innovadores de diseño y construcción. Trabajos recientes $(1,2)$ aportan información sobre su participación en el diseño estructural de edificios con hormigón armado, así como su participación en el desarrollo del sistema de construcción de forjados denominado «reticular celulado» o «RetCel» $(3,4)$ mientras que otras investigaciones $(5,6)$ sobre su vida y obra ofrece una visión mucho más panorámica de su trayectoria profesional y personal.

En efecto, Doménico Parma (Chiavari, 1920 - Bogotá, 1989) obtuvo su título de ingeniero en la Universidad de Génova y en 1946 emigró a Colombia donde desempeñó varios cargos en los ámbitos público y privado, antes de establecer su propia firma en 1962. A lo largo de sus 43 años de vida profesional en el país estuvo al frente del cálculo estructural de numerosos edificios y puentes; entre los primeros se destacan: sede del «Banco Central Hipotecario» (1962-64), «Avianca» (1965), «Torre Davivienda» (1965), «Residencias El Parque» (1965), «Seguros Tequendama» (1967) y «Telecom» (1972), a los que se suma el diseño del sistema de desplazamiento del edificio "Cudecom» (1973-74), este último reseñado en su momento en Informes de la Construcción (7). De sus diseños para puentes bien vale la pena mencionar el de Cambao sobre el río Magdalena (en servicio desde 1986), el puente sobre el río Chinchiná (en servicio desde 1991) y el puente de Anacaro, sobre el río Cauca (en servicio también desde 1991), además de su participación en el equipo a cargo de la construcción del puente «Alfonso López Pumarejo», sobre el río Magdalena, en cercanías de Barranquilla, diseñado por Ricardo Morandi (en servicio desde 1974).

Este artículo tiene como objetivo identificar, de manera más precisa el grado de participación de Parma en las fases de concepción y ejecución del último de sus proyectos de infraestructura civil (falleció cuando estaba al frente de la supervisión de su construcción): el puente sobre el río Chinchiná, bautizado de manera póstuma con su nombre, el cual no ha sido estudiado desde esta perspectiva de análisis (8).

Y es que en razón de la complejidad del lugar donde se tendría que levantar el puente, así como a los limitados recursos económicos y de tiempo, Parma tuvo que desarrollar varias propuestas de diseño para el puente, aplicando diferentes sistemas estructurales con el fin de que se pudiera evaluar en cada alternativa su factibilidad en términos de sus costos, cronograma y constructibilidad. En el presente trabajo se descifra, describe y analiza el proceso de diseño realizado por Parma, revisando el desempeño estructural de cada alternativa de diseño estudiada por él.

La investigación presentada aquí fue llevada a cabo utilizando la información disponible en el Archivo documental Doménico Parma en posesión de la Universidad de Los Andes y la revisión y análisis del poco conocido archivo de fotografías del hoy conocido como Puente Doménico Parma existente en el archivo de la Sociedad Colombiana de Ingenieros.

\section{EL PUENTE SOBRE EL RÍO CHINCHINÁ: EVOLUCIÓN Y DESARROLLO DEL PROYECTO}

Con motivo de la destrucción de un puente metálico existente sobre el río Chinchiná, causada por la avalancha que generó el deshielo del glaciar que cubría el volcán Nevado del Ruiz (erupción del 13 de noviembre de 1985), el Ministerio de Obras Públicas de Colombia convocó a una licitación para el diseño y construcción de un nuevo puente, mucho más durable.

Ganadora del proceso, la firma colombiana «Conconcreto» encargó a Parma el diseño del puente, que conforme al estudio de suelos debía asentarse en una zona caracterizada por la presencia de escorrentías de agua a escasa profundidad y bajas consistencias superficiales propias de terrenos sedimentarios, por lo que se recomendaban sistemas de cimentación profundos capaces de llegar $22 \mathrm{~m}$ por debajo del nivel previsible para otros más convencionales (9).

El trazado de la nueva vía a la que habría de servir el puente, incrementó la cota sobre el río cuyo cauce tenía un ancho aproximado de 60 metros, dejando además libre una zona inundable hacia la margen izquierda, con lo cual el puente proyectado debía cubrir una longitud total de aproximadamente $240 \mathrm{~m}$, con una altura sobre el cauce de entre $15 \mathrm{y}$ $25 \mathrm{~m}$.

En marzo de ese mismo año, Doménico Parma contaba ya con cuatro anteproyectos del puente que presentó a la firma «Conconcreto» y esta a su vez al Ministerio de Transporte, y aunque no es posible precisar el orden en que cada uno de ellos fue elaborado, sí es fácil advertir que todos respondían a un proceso de búsqueda formal y constructiva en procura de minimizar los costos y reducir al máximo el número de pilas sobre un terreno de difíciles condiciones geotécnicas (10).

Es importante precisar que la definición de la tipología de un puente depende de factores tales como la longitud total, la cantidad de apoyos y las dificultades del terreno, el trazado de la vía y la altura sobre el nivel medio de las aguas del río. Conceptualmente, en sitios donde la topografía y las condiciones de acceso son difíciles, es deseable tener la menor cantidad de apoyos sobre el suelo y resolver el tablero mediante una tipología que permita vencer las mayores luces posibles. Adicionalmente, la posibilidad de resolver constructivamente la estructura mediante elementos prefabricados y repetitivos, posibilita obtener mejores rendimientos en obra con mayor economía.

Por lo tanto, considerando que en todas las propuestas presentadas por Parma, el emplazamiento y las condiciones de carga son prácticamente las mismas, cada una de ellas intenta, desde una tipología diferente, dar respuesta a los factores mencionados. No se trata entonces de un mismo diseño que se transforma progresivamente en busca de la solución más acertada sino de diferentes alternativas concebidas en simultáneo por un único diseñador que finalmente las evalúa en función de variables tales como su costo, viabilidad constructiva (disponibilidad de medios materiales y capacidad de la mano de obra) y rapidez en la ejecución (dada la situación de emergencia que daba origen al proyecto).

Se presenta a continuación un análisis conceptual de las tipologías estructurales de cada alternativa, haciendo énfasis 
en los principios básicos de su funcionamiento estático como herramienta para entender sus ventajas y desventajas sin detenerse en los detalles del cálculo en cada caso. Sin embargo, en el apartado de este artículo dedicado a la Discusión de los resultados, se incluye la Tabla 1 que ayuda a entender la evaluación de las alternativas.

\subsection{Alternativa 1: puente de cuatro luces, con vigas de concreto y arco en acero}

En esta alternativa (11) se divide el puente en dos tipologías constructivas definidas: la primera mitad se resuelve mediante una estructura en concreto de tres tramos con apoyos en pilas intermedias de concreto separadas entre $40 \mathrm{~m}$ y 46 $\mathrm{m}$ sobre la zona de inundación del río, y la segunda mitad con una estructura en arco metálico que vence una luz de $120 \mathrm{~m}$ sobre el cauce del río (Figura 1).

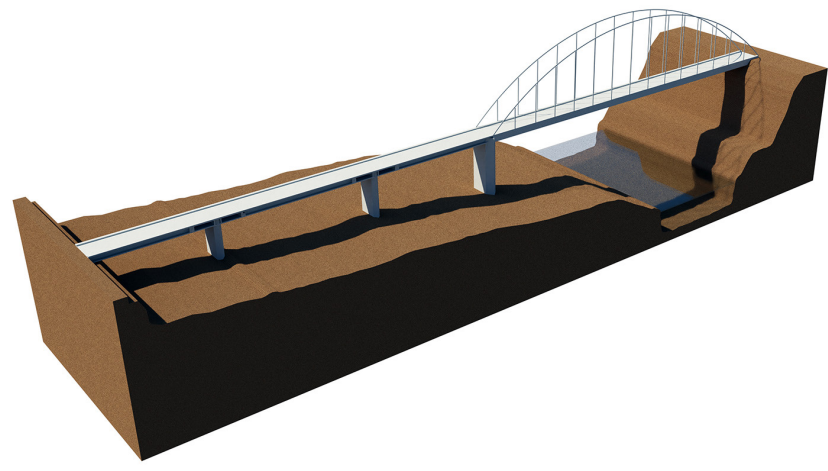

Figura 1. Anteproyecto de solución convencional con tramos rectos apoyados y arco metálico de gran luz. Fuente: elaboración propia a partir de (11).

El planteamiento estático del puente consiste en construir sobre las pilas intermedias una sección continua de tablero conformado por vigas cajón en voladizo, de 5,50 m de longitud a cada lado, y sobre ellas apoyar tramos simples de viga de 35 $m$ de luz (Figura 2). De esta manera se tipifican las luces y se resuelven con elementos modulares de vigas prefabricadas en concreto postensado para la sección del tablero. La luz final de $120 \mathrm{~m}$ se plantea mediante un tablero conformado por vigas metálicas apoyadas por arcos superiores también en acero. (a)
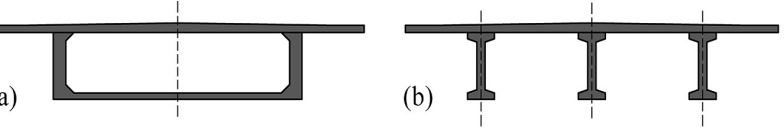

Figura 2. Sección de los tableros en la alternativa 1: (a) vigas cajón en voladizo con área de $3,58 \mathrm{~m}^{2}$ y (b) tramos simples con área de $3,20 \mathrm{~m}^{2}$. Fuente: elaboración propia a partir de (11).

Desde el punto de vista estructural, los tramos de viga suspendidos entre los voladizos de las pilas garantizan el funcionamiento como vigas simplemente apoyadas, generando el diagrama de momentos continuo para el puente con el punto de inflexión en los apoyos de los tramos suspendidos y el momento negativo sobre las pilas. El arco metálico, con pendolones cada $8 \mathrm{~m}$ aproximadamente, permite vencer la luz con un tablero más ligero y con solicitaciones por flexión de menor magnitud, ya que las fuerzas se transmiten mediante compresión a través del arco hacia los apoyos (Figura 3).

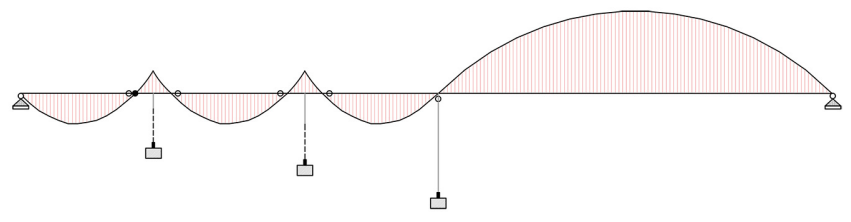

Figura 3. Geometría de la alternativa 1. Fuente: elaboración propia.

¿Por qué razón la propuesta fue desechada? Si bien es claro el comportamiento mecánico del sistema estructural, desde el punto de vista constructivo, esta alternativa presenta dos inconvenientes: la construcción de tres pilas de apoyo en el terreno, y la combinación de dos sistemas constructivos: el tablero de concreto y la estructura metálica del arco. El mayor número de apoyos en el terreno implica un trabajo de excavación y cimentación más complejo, que dadas las condiciones topográficas y del subsuelo implicaban una mayor duración de las obras. En cuanto al tablero del puente, si bien el tramo en concreto se resuelve mediante una sección típica de tres vigas postensadas y losa de concreto vaciado en sitio, la fabricación e instalación del arco metálico requiere de diferentes procesos y equipos a los utilizados en la primera mitad de la estructura, con lo cual se hubieran requerido dos contratistas distintos. La ventaja del arco con-

Tabla 1. Comparación de criterios de cada alternativa de solución para el puente.

\begin{tabular}{|c|c|c|c|c|}
\hline Criterio & Alternativa 1 & Alternativa 2 & Alternativa 3 & Alternativa 4 \\
\hline $\begin{array}{l}\text { Afectación del cauce por } \\
\text { apoyos intermedios }\end{array}$ & Alta & Media & Mínima & Baja \\
\hline $\begin{array}{l}\text { Posibilidad de prefabricación } \\
\text { de la sección }\end{array}$ & Media & Baja & Total & Total \\
\hline Peso propio del tablero (t) & 1590 & 2625 & 1910 & 1850 \\
\hline $\begin{array}{l}\text { Altura de la sección (vigas + } \\
\text { losa, en metros) }\end{array}$ & $1.80 \mathrm{~m}$ & $\begin{array}{l}\text { Variable, entre } 1.80 \mathrm{y} \\
7.00 \mathrm{~m}\end{array}$ & $1.80 \mathrm{~m}$ & $1.70 \mathrm{~m}$ \\
\hline Rigidez torsional & $\begin{array}{l}\text { Baja. La sección del } \\
\text { tablero es abierta (vigas } \\
\text { prefabricadas y losa } \\
\text { superior) }\end{array}$ & $\begin{array}{l}\text { Solo en sección variable. } \\
\text { Los tramos suspendidos } \\
\text { son en sección abierta }\end{array}$ & Alta. Sección en cajón. & Alta. Sección en cajón. \\
\hline Deflexiones & Menor a L/1000 & $\mathrm{L} / 1000$ & $\mathrm{~L} / 800$ & $\mathrm{~L} / 800$ \\
\hline $\begin{array}{l}\text { Especialización en mano de } \\
\text { obra y equipos }\end{array}$ & $\begin{array}{l}\text { Media. Dos tipos de } \\
\text { tecnología: concreto } \\
\text { preesforzado y estructura } \\
\text { metálica }\end{array}$ & $\begin{array}{l}\text { Alta. Tecnología de } \\
\text { concreto preesforzado y } \\
\text { voladizos sucesivos. }\end{array}$ & $\begin{array}{l}\text { Alta. Prefabricación, } \\
\text { izaje y montaje de tablero } \\
\text { colgante y anclajes a la } \\
\text { roca. }\end{array}$ & $\begin{array}{l}\text { Media. Prefabricación, } \\
\text { izaje y montaje de tablero } \\
\text { colgante. }\end{array}$ \\
\hline $\begin{array}{l}\text { Necesidad de mantenimiento } \\
\text { durante operación }\end{array}$ & Alta: estructura en acero. & Baja & $\begin{array}{l}\text { Alta: cables y pendolones } \\
\text { de acero. }\end{array}$ & $\begin{array}{l}\text { Alta: cables y pendolones } \\
\text { de acero. }\end{array}$ \\
\hline
\end{tabular}


siste en lograr un menor peso propio para la superestructura, que para este caso tendría un peso propio aproximado de 1.590 toneladas.

\subsection{Alternativa 2: puente continuo de tres luces en concreto}

En esta alternativa se elimina uno de los apoyos intermedios, con el consecuente aumento en la magnitud de las luces. Se trata de tres luces de 42,75 m - 92,75 m y $97 \mathrm{~m}$, manteniendo la tipología de tramos suspendidos de $35 \mathrm{~m}$ de longitud entre pilas con cabezales en voladizo (Figura 4).

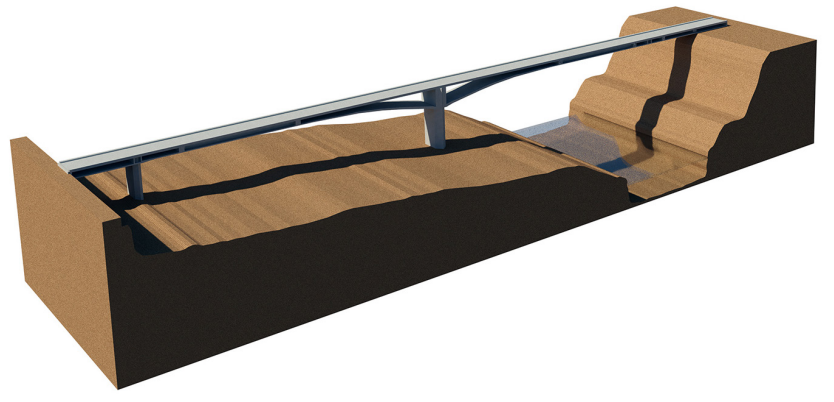

Figura 4. Anteproyecto de voladizos balanceados. Fuente: elaboración propia a partir de (11).

La pila central soporta una sección en cajón que se proyecta en voladizos compensados de $50 \mathrm{~m}$ a cada lado, con una sección variable desde $7 \mathrm{~m}$ de altura en el apoyo hasta 1,85 $\mathrm{m}$ en el extremo, donde se apoya el tramo suspendido típico (Figura 5). En este planteamiento la totalidad de la estructura se resuelve en concreto postensado, siendo la pila central y los voladizos compensados el componente principal de la solución debido a la magnitud de las cargas que recibe, $\mathrm{y}$ al proceso constructivo requerido mediante la tecnología de voladizos sucesivos para la construcción de la sección en cajón de altura variable.

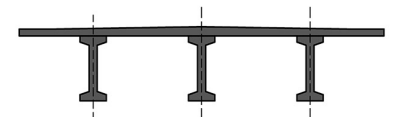

(a)

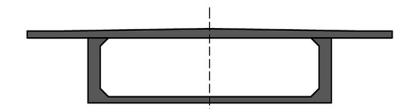

(b)

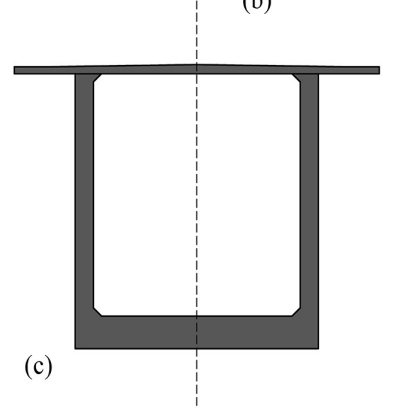

Figura 5. Sección variable del tablero en la alternativa 2: (a) tramos suspendidos con área de $3,204 \mathrm{~m}^{2}$, (b) sección inicial en voladizo con área de $3,581 \mathrm{~m}^{2}$ y (c) sección final en voladizo con área de $11,988 \mathrm{~m}^{2}$. Fuente: elaboración propia a partir de (11).

Las razones que motivan el rechazo de esta propuesta se encuentran seguramente en la ineficiencia del sistema, entendida como la relación entre resistencia efectiva y peso propio: como se aprecia en el diagrama de momentos general del puente (Figura 6), la definición de la pila central con los voladizos de 50 m genera un momento negativo en el apoyo de gran magnitud, que requiere de una sección de $7 \mathrm{~m}$ de altura equivalentes a 1/7 de la luz. El tamaño de la sección de la viga cajón hace que el peso propio del tablero del puente sea mayor, incidiendo directamente en el tamaño del cimiento que debe ser mayor si se compara contra la primera alternativa, al menos para la pila central.

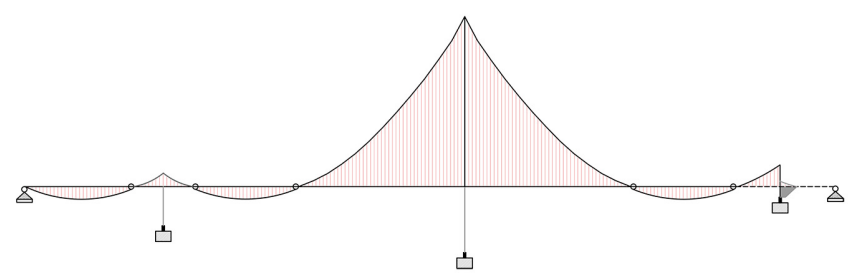

Figura 6. Diagrama de momentos por carga muerta para la alternativa 2. Fuente: elaboración propia.

Por otra parte, la construcción de la pila central y los voladizos compensados se hace mediante armado y vaciado en sitio de cada tramo, limitando el uso de elementos prefabricados a los tres tramos suspendidos de $35 \mathrm{~m}$ de luz. En general, esta es la alternativa con el mayor peso propio por las dimensiones de las vigas del tablero, alcanzando un valor aproximado de 2.620 toneladas.

\subsection{Alternativa 3: puente colgante de una luz}

Con el criterio de minimizar el número de apoyos en el terreno, la alternativa de una estructura que pueda vencer los $240 \mathrm{~m}$ de luz sobre el cauce del río se resuelve mediante un puente colgante. Para esta opción, Parma propone una configuración de puente colgante con tramo central de $240 \mathrm{~m}$, una luz lateral hacia el margen izquierdo de $80 \mathrm{~m}$ para compensar el cable parabólico principal, y hacia el margen derecho el anclaje directamente a la montaña, de modo que se elimina la necesidad de construir un segundo pilón o torre (Figura 7).

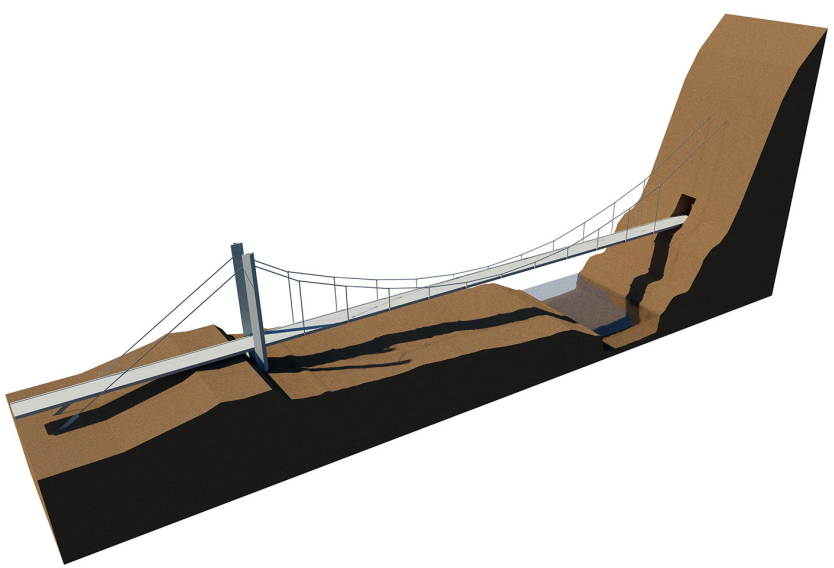

Figura 7. Anteproyecto de puente tipo colgante con mástil único en un extremo y anclaje opuesto en roca.

Fuente: elaboración propia a partir de (11).

La ubicación de la torre en la zona menos profunda, al inicio del vano, permite tener un elemento de menor altura y la posibilidad de construir sobre relleno la primera luz del puente, de modo que solamente el tramo central de tablero está suspendido. El tablero del puente, conformado por una viga cajón de dos celdas, se conforma mediante tres vigas prefabricadas de concreto de sección en «T», y la losa superior e inferior vaciadas en sitio. Las vigas se sujetan al cable mediante vigas transversales cada $20 \mathrm{~m}$, con lo cual las dimensiones de la estructura son menores y repetitivas. 
Como ventaja de esta solución con relación a las dos anteriormente expuestas, aquí se requiere la construcción de un solo apoyo en el terreno, presentando la menor intervención en el cauce, y permite la prefabricación total del tablero con tramos de viga de la misma longitud. Sin embargo, su dificultad está en la propuesta de anclaje directamente contra la roca en el extremo derecho la cual requiere de la construcción de un túnel para cada cable, de manera que se penetre la superficie de la roca y se puedan realizar las perforaciones para instalar tensores o tie-backs que desarrollen la suficiente capacidad a tracción para estabilizar el cable principal. Esta condición requiere de una investigación geotécnica más profunda y localizada en la parte alta de la montaña donde se propone el anclaje, con lo cual el tiempo de desarrollo delos estudios se prolonga y la ejecución de labores en obra es compleja debido a la ubicación de los anclajes, la excavación del túnel y la incertidumbre respecto de la capacidad real de los anclajes, que solamente se puede verificar una vez se ejecuta y se realizan pruebas de carga al mismo.

Aun cuando la solución colgante proporciona ventajas en términos de la construcción de cimentación y apoyos, así como la posibilidad de prefabricar el tablero del puente, la necesidad de un relleno por detrás del margen izquierdo a la entrada de puente y la propuesta de anclaje del cable principal directamente a la roca mediante túneles y perforaciones conllevan imprevistos de obra difíciles de prever y por tanto posibles sobrecostos y demoras para el proyecto, que hicieron que esta alternativa tampoco fuera adoptada.

\subsection{Alternativa 4: puente colgante de dos luces}

A partir del planteamiento del puente colgante tradicional, Parma plantea la solución que al final resulta siendo la opción definitiva: aprovechando las ventajas del tablero colgado, decide utilizar una única pila central de la que suspende un cable que se ancla en cada extremo del puente, venciendo la longitud total mediante dos luces simétricas de 120 m (Figura 8).

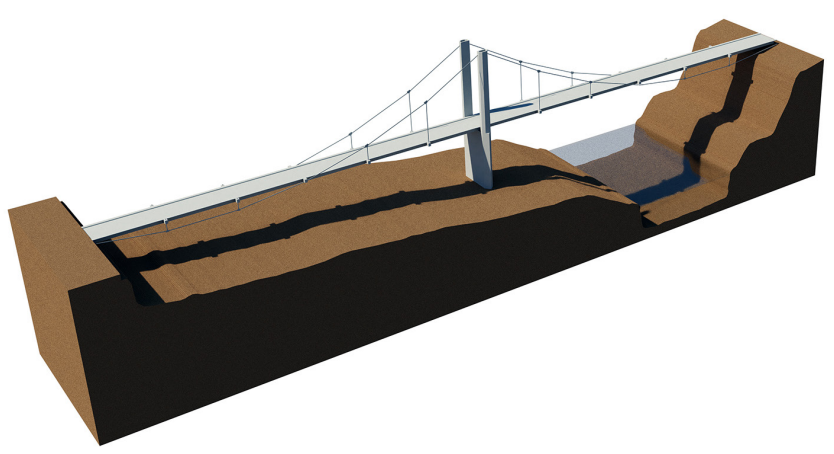

Figura 8. Anteproyecto con la solución finalmente adoptada. Fuente: elaboración propia a partir de (11).

Esta solución sintetiza las ventajas de cada una de la alternativas estudiadas, llegando además a una solución estática y formal poco común y muy eficiente: el trazado del cable para el puente colgante sigue la geometría del diagrama de momentos de la pila central con los tramos en voladizo a ambos lados desarrollado en la opción 2, y permite suspender mediante pendolones cada $20 \mathrm{~m}$ los tramos de tablero que se conforman mediante vigas prefabricadas, obteniendo así el menor peso propio para la superestructura y la posibilidad de tipificar los componentes de la construcción y obtener las ventajas de tiempo y montaje que se requerían para el proyecto (Figura 9).

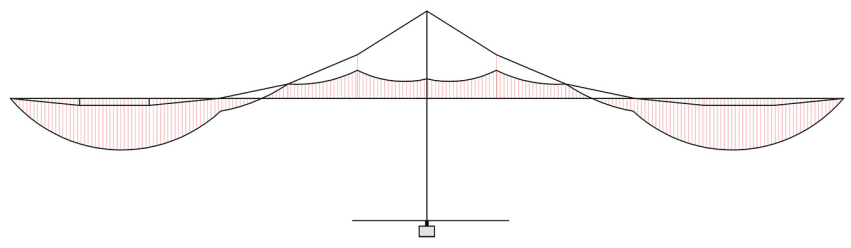

Figura 9. Diagrama de momentos flectores por carga muerta en la alternativa 4.

Fuente: elaboración propia.

Adicionalmente el planteamiento estructural del puente mejora las condiciones de llegada a los extremos respecto de la alternativa colgante tradicional: al pasar el cable por debajo del tablero en la llegada a los extremos, se evita el relleno por detrás de los estribos y se puede instalar el macizo de anclaje a nivel de la rasante. Esta característica es atípica en los puentes colgantes, ya que la mayoría de las veces el tablero se encuentra siempre por debajo del cable, o a lo sumo anclando su punto más bajo en el centro de la luz.

Al tener el tablero soportado por los pendolones, y considerando la continuidad entre la pila central y la viga cajón del puente, el trazado del cable principal se puede entender conceptualmente a partir de la superposición del diagrama de momentos para una viga en voladizo y una viga simplemente apoyada, cada una recibiendo las cargas puntuales que generan las reacciones de los pendolones en cada tercio de la luz. Los diagramas de momento (Figuras 10 y 11) ilustran la lógica estructural del funcionamiento del puente.

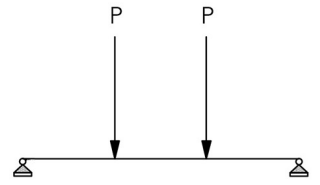

$\mathrm{L} / 2$

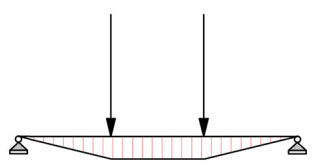

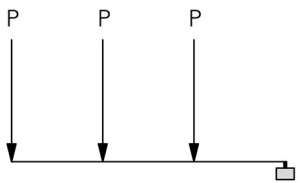

$\mathrm{L} / 2$

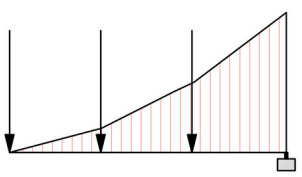

Figura 10. Interacción de la viga simplemente apoyada (izquierda) y la viga en voladizo (derecha) con sus respectivos diagramas de momentos generados por las cargas puntuales a L/3. Fuente: elaboración propia.

Del diagrama de momentos de vigas simples:
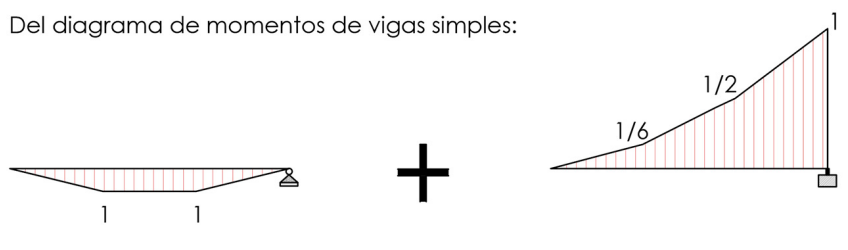

...al trazado del cable de suspensión del puente.

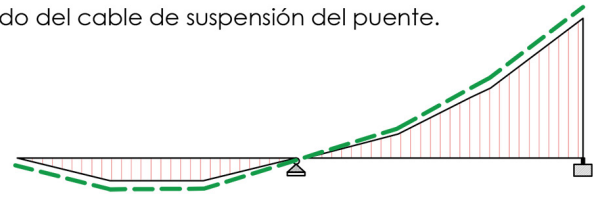

Figura 11. Trazado del cable principal de medio puente como resultado de la adición de la estática de cada una de las partes del tablero. Fuente: elaboración propia. 
Con esta configuración se tienen dos pendolones a tracción a cada lado del mástil central, y dos puntales a compresión bajo las vigas del tablero en los vanos cercanos a los extremos del puente. El hecho de pasar el cable por debajo del tablero permite tener una altura menor en la torre y mejorar la estabilidad lateral del puente por el efecto de la reacción vertical ascendente generada en los "puntales" (Figura 12).

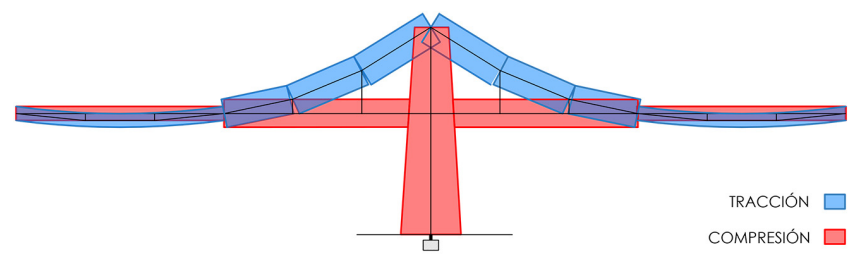

AXIALES POR CARGA MUERTA

Figura 12. Diagrama de fuerzas axiales en el puente. Fuente: elaboración propia.

La separación de los pendolones cada 20 metros permite además tener una sección total del tablero de menor altura respecto de las demás alternativas analizadas. En este caso, el tablero completo tiene un canto de $1.70 \mathrm{~m}$, correspondiente a 1/70 de la luz entre apoyos, relación que se encuentra dentro de los límites recomendados para la altura de las vigas de rigidez en estructuras colgantes (12).

La sección transversal del tablero se cuelga de los cables principales mediante vigas transversales, también en concreto postensado, que garantizan la transmisión de carga de las vigas longitudinales a los pendolones y proporcionan rigidez torsional a la sección del puente (Figura 13). La decisión de conformar una sección cerrada en cajón fundiendo una losa inferior que vincula los patines inferiores de las vigas prefabricadas le otorga un mejor comportamiento al tablero ante cargas excéntricas que generan torsiones, como efectivamente sucede cuando transita una carga pesada por uno de los carriles de la vía.

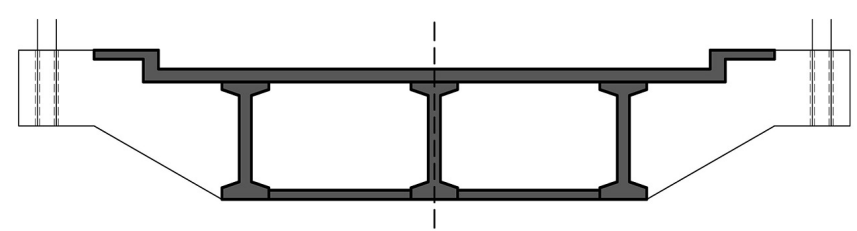

Figura 13. Sección del tablero en la alternativa 4, con área de 3,215m². Fuente: elaboración propia a partir de (11).

Así, desde el punto de vista mecánico, el puente quedaba integrado por dos sistemas independientes pero que interactuaban entre sí, por lo que ambos presentan compatibilidad de deformación. Los dos sistemas fueron denominados por el propio Parma (13) como «sistema pórtico» y «sistema catenaria» (Figura 14): el primero estaba conformado por la torre central y las vigas de rigidez longitudinales, de tal manera que:

- El mástil central «CE» se considera empotrado en «C» (que corresponde a la cimentación profunda mediante caissons).

- Se tienen dos claros de igual luz «S».

- Existe continuidad estructural entre todos los miembros en el punto «D».

- Las vigas dispuestas en los sentidos « $\mathrm{AD}$ » $\mathrm{y}$ «DB» tienen apoyos simples en sus extremos «A» $\mathrm{y}$ «B», respectivamente, pero movimiento restringido en el sentido vertical.

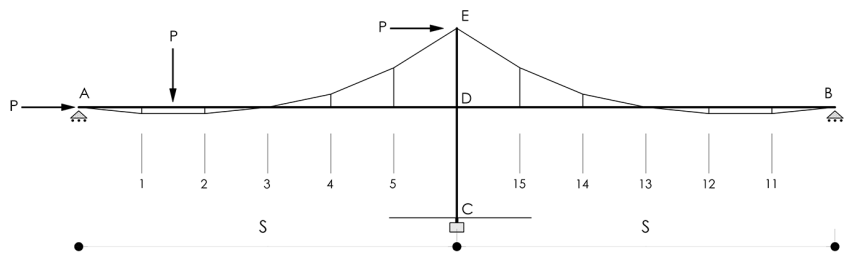

Figura 14. Abstracción de los dos sistemas estructurales del puente: el «sistema pórtico» y el «sistema catenaria». Fuente: elaboración propia.

Por su parte, el sistema «catenaria» estaba formado por la propia catenaria más los 10 pares de pendolones en suspensión.

\section{DISCUSIÓN}

No es posible saber con exactitud cuáles fueron los criterios de los que se valió Parma (y casi con seguridad la empresa «Conconcreto», responsable del contrato de diseño y construcción) pues no ha quedado registro de ello; sin embargo, un análisis por comparación de ocho factores de interés (Tabla 1) puede ayudar a comprenderlo.

La afectación al cauce del río es baja en la opción escogida, lo cual minimiza el riesgo frente a nuevas crecidas del río; además, concentra las labores de cimentación en un solo punto y reduce al máximo la dedicación a las labores de construcción del mástil.

De otra parte, dado el interés de Parma por la prefabricación y la industrialización de los procesos de ejecución (demostrado a lo largo de toda su labor como ingeniero) es notoria la manera en que la alternativa 4 permite un ejercicio de la construcción del puente a partir de piezas industrializadas de acero (cables, pendolones, puntales y abrazaderas) y elementos de concreto prefabricado en el sitio (vigas y prelosas para el tablero), con la consecuente reducción del plazo de ejecución y disminución del precio final del proyecto.

Desde el punto de vista mecánico, la propuesta que finalmente se construyó es la que logra un adecuado balance entre el peso propio y la altura de la sección en las vigas del tablero, sin detrimento de la rigidez torsional, aunque con mayores deflexiones que en las alternativas restantes.

Sin embargo, de nuevo razones de tipo constructivo (especialización en mano de obra y equipos, así como necesidad de mantenimiento durante la operación), ponen énfasis en considerar a la última de las alternativas como la más conveniente dadas las características generales del proyecto.

Así, pese a que el requerimiento de un puente de longitud total superior a los 200 metros implica, en cualquier caso, una solución estructural y constructiva compleja, la opción colgante con torre central propuesta por Parma resultó eficiente técnica y económicamente para el proyecto, y pudo ser llevada a cabo gracias a que el alcance del consultor no se limitó únicamente a los planos y memorias de diseño, sino que incluyó el estudio y análisis del proceso constructivo y de montaje de la estructura.

También es importante mencionar que el cálculo estructural se desarrolló a partir del marco normativo de la AASHTO de $1983(14,15)$ con especial consideración a los aspectos sís- 
micos dadas las condiciones de la región y del país que para entonces solo contaba con un código en la materia (16). Es destacable además la utilización de herramientas informáticas de análisis creadas por el mismo diseñador para acometer el cálculo de las diferentes condiciones del cable bajo carga: «CATE» que suministraba la geometría de la catenaria en función de la longitud de los segmentos, las cargas en los nudos, el claro y la diferencia de nivel entre anclajes; «DEF» que suministraba las deformaciones en todos los puntos críticos del pórtico sin las catenarias; «GEO» que suministraba las nuevas coordenadas de los nudos después de haber ocurrido las deformaciones y «CHINA» por el cual se determinaba el cálculo general integrando las condiciones extremas como podía ser el corte de un pendolón.

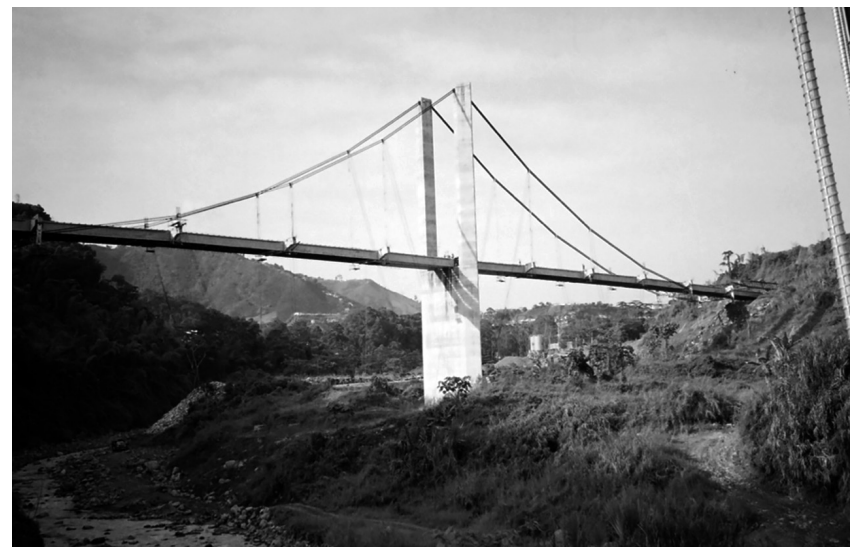

Figura 15. El puente Doménico Parma, en la actualidad. Fuente: propia.

Para efectos de la carga sísmica, Parma consideró fuerzas en sentido longitudinal siguiendo las recomendaciones de la AASHTO (14) asumiendo que el 100\% de la carga horizontal equivalente de todo el puente era aplicada a nivel de calzada (13). Por su parte, en sentido transversal Parma asumió fuerzas horizontales transversales equivalentes actuando a nivel del centroide de la calzada (362 toneladas en torre y 181 toneladas en los estribos).

A lo largo de sus 30 años de vida útil, el puente ha demostrado su capacidad de resistencia a los numerosos eventos sísmicos que han afectado la región en donde se levanta (Figura 15).

\section{CONCLUSIONES}

El estudio y análisis del proceso llevado a cabo por Doménico Parma para el diseño definitivo para el puente sobre el rio Chinchiná, ilustra muy bien el papel fundamental que él tuvo, liderando el equipo de profesionales que finalmente lograron, a pesar de su ausencia en el final del proceso, construir de manera segura, con costos controlados y en poco tiempo el puente que de manera urgente necesitaba la región.
El éxito final, en el proyecto, es fácilmente atribuible a la manera como abordó Parma el proceso de diseño: mediante la elaboración casi completa de diferentes alternativas que permitían evaluar rápidamente costos, tiempos, desempeño estructural e imagen estética de cada una de las propuestas.

La labor de Parma desde las primeras etapas, con el planteamiento del problema, el análisis de diferentes alternativas de solución, y la definición y refinamiento del diseño elegido, es un ejemplo del pensamiento innovador del consultor y del compromiso con los valores de eficiencia técnica, constructiva y económica que hacen parte de la práctica del diseño en ingeniería.

$\mathrm{Al}$ revisar formalmente las alternativas propuestas por Parma para la geometría del puente, verificando sus notables diferencias, se concluye que el diseñador procuró elaborar con cierto grado de resolución suficiente, una amplia gama de opciones, de tal manera que la tarea de decisión resultara más completa.

Del análisis de las diferentes alternativas propuestas por Parma, para la solución a un puente de alrededor de $200 \mathrm{~m}$ de luz, en unas condiciones de lugar muy complicadas, se concluye que decisión respecto a la alternativa escogida, pasó por la evaluación de múltiples variables, entre las que mediante el presente trabajo, se pudieron evidenciar y valorar el desempeño de las siguientes: la menor afectación posible del cauce del rio, las posibilidades para prefabricar la mayor parte de los elementos de la sección del tablero así como el peso propio y altura resultante del tablero. Igualmente, la rigidez torsional de cada alternativa, destacándose la alternativa finalmente escogida por contar con una sección en cajón que jugaría un papel protagónico durante el episodio de falla de uno de los pendolones. La necesidad de implementación de procesos de obra complejos, en términos de equipos, formaletas aéreas, etc.

Por último, la propuesta escogida y finalmente construida, mediante su definición a través de un cable aparentemente catenario, pero que luego del análisis, se pudo identificar que se aproxima de manera notable en su geometría al diagrama de momentos al que se verá sometida la estructura. Adicionalmente, el paso del cable principal por debajo del tablero al llegar a cada aproche, muestran un diseño que en términos tipológicos resulta innovador para el momento y contexto local.

La tipología de puente colgante con un único mástil central sigue siendo única, y al día de hoy no se tiene registro de puentes similares construidos en Colombia en los años posteriores. Por el contrario, la ingeniería local fue dejando de lado cualquier tipo de ejercicio experimental, limitándose a la aplicación de un repertorio tipológico conocido y bien aceptado pero que en cualquier caso, demanda del previo reconocimiento de las condiciones propias de cada lugar. 


\section{REFERENCIAS}

(1) Vargas, H. (2008). El desarrollo de la edificación en concreto armado en Colombia: el caso de los pioneros Doménico Parma y Guillermo González Zuleta (1945-1985). DeArq, 04(1): 64-75. https://doi.org/10.18389/dearq4.2009.09

(2) Villate, C. (2008). Edificios en altura. La carrera técnica por ganar en el skyline. El caso colombiano: Doménico Parma. DeArq, 03(1): 61-66. https://doi.org/10.18389/dearq3.2008.08

(3) Vargas, H. (2012). RetCel: The Development of Floor and Roof Assemblies of Precast Concrete Cells in Colombia: 1949-1989. En R. Carvais et al. (Eds.), Nuts \& Bolts of Construction History. Culture, Technology and Society (vol. 3, pp. 431-438). París: Piccard.

(4) Cortés, E.C. y Primmer, A. (2013). Doménico Parma and Guillermo González Zuleta: a story of challenges, innovations and development of concrete architecture in Colombia. En P. Cruz (Ed.), Structures and Architecture: Concepts, Applications and Challenges (pp. 1907-1914). Guimaraes: CRC Press.

(5) Varini, C. (2004). Doménico Parma. Retrato científico. Bogotá: Universidad Católica de Colombia.

(6) Giovannardi, F. y Parma, A. (2019). Domenico Parma. Ingegnere italiano a Bogotá. Vite e opere. Roma: Ed. Aracne.

(7) Páez, A. (1975). Traslado del edificio Cudecom. Bogotá, Colombia. Informes de la construcción, 27(274): 49-64. https:// doi.org/10.3989/ic.1975.v28.i274.2832

(8) Villate, C. (2017). El puente Doménico Parma (Colombia): adaptación de innovaciones en procesos constructivo de estructuras de cables. En S. Huerta et al. (Eds.), Actas del Décimo Congreso Nacional y Segundo Congreso Internacional Hispanoamericano de Historia de la Construcción (vol. III, pp. 1737-1745). Madrid: Instituto Juan de Herrera.

(9) Orozco, L.F. (1986). Estudio de suelos y análisis de cimentaciones puente Chinchiná - Chinchiná- Caldas. Bogotá: Manuscrito Archivo Parma, caja 143, Universidad de los Andes.

(10) Galvis, J. (28 de febrero de 2018). Rehabilitación Puente Parma. (C. Villate, N. Parra, \& A. Posada, entrevistadores). Bogotá.

(11) Parma, D. (1986). Puente de Chinchiná. Diseños Bogotá: Manuscrito Archivo Parma, caja 140-142, Universidad de los Andes.

(12) Gimsing, N. (1983). Cable Supported Bridges: Concept and design. New York: John Wiley.

(13) Parma, D. (1987). Puente de Chinchiná. Memoria de cálculo (1 ${ }^{a}$ y $2^{a}$ parte). Bogotá: Manuscrito Archivo Parma, caja 145, Universidad de los Andes.

(14) AASHTO (1983). Standard Specifications for Highway Bridges (Ed. rev.). Washington: AASHTO.

(15) AASHTO (1983). Guide Specifications for Seismic Design of Highway Bridges. Washington: AASHTO.

(16) AIS (1984). Código colombiano de construcciones sismo - resistentes. Bogotá: Asociación Colombiana de Ingeniería Sísmica. 